\title{
Metodologías innovadoras y Tecnologías del Aprendizaje y el Conocimiento (TAC) en la materia Didáctica de la Educación Física en el Grado de Ciencias de la Actividad Física y el Deporte de la FPCEE Blanquerna - Universidad Ramon Llull
}

\section{Dr. Josep Campos-Rius ${ }^{a}$ y Dr. Enric M. Sebastiani Obrador ${ }^{b}$}

${ }^{a}$ Grupo de Investigación e Innovación sobre Deporte y Sociedad (GRIES), FPCEE Blanquerna Universidad Ramon Llull (josepcr@blanquerna.edu), ${ }^{a}$ Grupo de Investigación e Innovación sobre Deporte y Sociedad (GRIES), FPCEE Blanquerna - Universidad Ramon Llull (enricmariaso@blanquerna.url.edu).

\begin{abstract}
It presents a teaching experience of innovation and the use of Learning and Knowledge Technologies (LKT) within the framework of the subject matter of Physical Education of the university degree in Sciences of Physical Activity and Sport in the FPCEE Blanquerna - Ramon Llull University.

The development of digital competence and on-demand methodology is considered as a variant of Project-Based Learning, aimed at enhancing student proactivity in the acquisition of competencies, knowledge and skills, specifically in the use of new technologies in the teaching.

The activities described are specified in the use of Audiovisual Media and LKT; the presentation of the content of the subject by the students in small groups; and the participation in the organization of a gamified sport promotion activity from School Physical Education with real students.

The assessment of the satisfaction and the use of the subject by the students is analyzed from their opinion through a questionnaire at the end of the course and through the different proposals for innovation and the use of technology that they carry out during the subject.
\end{abstract}

Keywords:

Digital competence, Information and Communication Technologies (ICT), Learning and Knowledge Technologies (LKT), Physical Education, projectbased learning

\section{Resumen}

Se presenta una experiencia docente de innovación y del uso de las Tecnologías del Aprendizaje y el Conocimiento (TAC) en el marco de la materia Didáctica de la Educación Física del Grado universitario en Ciencias 
Implementación de las Tecnologías de la Información y la Comunicación (TAC) en la materia Didáctica de la Educación Física en el Grado de Ciencias de la Actividad Física y el Deporte de la FPCEE Blanquerna - Universidad Ramon Llull

de la Actividad Física y el Deporte en la FPCEE Blanquerna - Universitat Ramon Llull.

Se plantea el desarrollo de la competencia digital y la metodología ondemand, considerada como una variante del Aprendizaje Basado en Proyectos, orientada a potenciar la proactividad del alumno en la adquisición de competencias, conocimientos y habilidades, concretamente en el uso de nuevas tecnologías en la enseñanza.

Las actividades descritas se concretan en el uso de Medios Audiovisuales (MAV) y las TAC; la presentación de contenidos de la materia por parte de los alumnos en pequeños grupos; y la participación en la organización de una actividad gamificada de promoción del deporte desde la Educación Física escolar con estudiantes reales.

La valoración de la satisfacción y el aprovechamiento de la materia por los estudiantes se analiza desde su opinión mediante un cuestionario al final del curso y a través de las diferentes propuestas de innovación, y del uso de la tecnología que llevan a cabo en la materia.

Palabras clave: Competencia digital, Tecnologías de la Información y la Comunicación (TIC), Tecnologías del Aprendizaje y del Conocimiento (TAC), Educación Física, Aprendizaje Basado en Proyectos (ABP)

\section{Introducción}

El manejo de las tecnologías y los MAV por parte del profesorado es un elemento y el dominio de diferentes metodologías y estrategias de enseñanza son elementos sensibles e importantes en el paradigma educativo de la actualidad, sobre todo desde la perspectiva de la formación basada en competencias.

La legislación educativa plantea que las competencias necesarias para el uso de las tecnologías tanto en la educación primaria como en la educación secundaria obligatoria se deben desarrollar en el nivel adecuado y se agrupan en diferentes ámbitos como son instrumentos y aplicaciones (1), el tratamiento de la información y organización de los entornos de trabajo y aprendizaje (2), la comunicación interpersonal y colaboración (3), y la ciudadanía, hábitos, civismo e identidad digital (4).

El logro de la competencia digital se ve favorecida por los aprendizajes significativos y motivadores para los alumnos que las tecnologías móviles hacen posible (Consell Escolar de Catalunya, 2015).

Se nos presenta un nuevo paradigma, el de ajustar la enseñanza y el aprendizaje restando importancia a la memorización, ya que Internet nos permite acceder al conocimiento y 
almacenarlo i, a cambio, estimular la creatividad, la comprensión de ideas y el pensamiento crítico (Reig, 2012; Reig y Vílchez, 2013). Tal y como nos plantea Ferreras (2011), esta visión debe tener en cuenta como complemento y apoyo a las diferentes actividades planteadas en las sesiones Educación Física tanto dentro como fuera de éstas. También destaca que la actual desmotivación y desinterés de los estudiantes por los contenidos teóricos $\mathrm{y}$, en algunos casos, procedimentales de las materias hace imprescindible que el área de Educación Física utilice herramientas tecnológicas que los estudiantes utilizan diariamente fuera de la escuela.

El área de Educación Física debe incorporar el nuevo paradigma educativo desde una perspectiva metodológica y didáctica, para el análisis de la acción docente, para el intercambio de información, conocimiento y experiencias entre el profesorado, el alumnado y entre ellos mismos (Campos-Rius y Sebastiani, 2016).

Para Arévalo (2016), la Educación Física tiene como principal eje el movimiento humano y será cuerpo (entendido como ser en sí mismo) el medio y la finalidad al mismo tiempo. Por este motivo, en la Educación Física los recursos tecnológicos tienen la misión de facilitar el aprendizaje y el desarrollo de las capacidades, siendo instrumentos, en cualquier caso, que no sustituyen la necesaria experiencia corporal vital que el estudiante debe tener en esta educación.

Ferreres (2011) destaca que la actual desmotivación y falta de interés por parte del alumnado ante los contenidos teóricos y, en algunos casos, procedimentales de la asignatura hace imprescindible que el área de educación física emplee para la enseñanza aquellas herramientas tecnológicas que el alumnado utiliza diariamente fuera de la escuela y que permiten que se sienta más interesado por los contenidos, tanto teóricos como prácticos, de la asignatura.

En relación a las posibilidades de incorporar las TIC en la Educación Física, éstas no pueden ni deben sustituir la actividad motriz en la escuela, sin embargo, se presentan como una buena herramienta para fomentar actitudes y procesos de reflexión por parte del alumnado (Capllonch, 2005).

Diversos autores han planteado la necesidad de formación en nuevas y diferentes metodologías de enseñanza-aprendizaje del profesorado de Educación Física, de la formación inicial de grado a los másteres universitarios de formación del profesorado de Educación Física de las Etapas Secundaria, Bachillerato, Ciclos Formativos y formación de técnicos deportivos (Carreiro, González y González, 2016; López, Pérez, Manrique y Monjas, 2015; Peire y Estrada, 2015; Lleixà, 2017).

\section{Objetivos}

El trabajo se enmarca en el tercer curso del Grado en Ciencias de la Actividad Física y el Deporte (CAFE) de la Facultad de Psicología, Ciencias de la Educación y el Deporte (FPCEE) Blanquerna - Universitat Ramon Llull (URL) y tiene como objetivos: 
Implementación de las Tecnologías de la Información y la Comunicación (TAC) en la materia Didáctica de la Educación Física en el Grado de Ciencias de la Actividad Física y el Deporte de la FPCEE Blanquerna - Universidad Ramon Llull

\section{Objetivos generales:}

- Desarrollar la competencia digital

- Fomentar el uso de las TIC y las TAC y los MAV

\section{Objetivos específicos}

- Reconocer metodologías de enseñanza-aprendizaje innovadoras con el uso de las TAC y los MAV y su aplicación práctica

- Estimular aprendizajes significativos y relevantes

\section{Desarrollo de la innovación}

El profesorado responsable de este proyecto está formado por los dos profesores responsables de la materia del Grado en Ciencias de la Actividad Física y el Deporte en los turnos de mañana y tarde, que se cursa en el segundo semestre del tercer curso académico. Estos profesores han diseñado, coordinado e implantado el proyecto educativo a desarrollar en la materia y han ido actualizando las herramientas metodológicas año a año (Campos-Rius y Sebastiani, 2016).

Desde la implantación del Grado en Ciencias de la Actividad Física y el Deporte se ha fomentado el desarrollo e implantación de nuevas y diferentes metodologías docentes y la contribución a la competencia digital orientando a los alumnos para que ellos mismos las puedan utilizar en su ejercicio profesional como docentes.

En el desarrollo de la experiencia se parte de la metodología on demand (Canaleta, Vernet y Navarro, 2013) que puede ser considerada una variante del Aprendizaje Basado en Proyectos (Project-based Learning) para potenciar la proactividad del alumno en la adquisición de competencias, conocimientos y habilidades, concretamente, en el uso de nuevas tecnologías en la enseñanza.

Se concibe el Aprendizaje Cooperativo Basado en Proyectos (ACBP) como método de trabajo y la introducción de las TIC y las TAC y la innovación dentro del contenido curricular de la didáctica Educación Física.

También se usa la metodología Flipped Classroom, que transfiere el trabajo de determinados procesos de aprendizaje fuera del aula y utiliza el tiempo de clase, junto con la experiencia del docente, para facilitar y potenciar otros procesos de adquisición y práctica de conocimientos dentro del aula.

En la materia se plantea la evaluación pura por competencias aportando retroalimentación formativa mucho más enriquecedora y significativa.

Se plantea la materia con el objetivo de construir criterios educativos sólidos y tomar conciencia de la función transformadora de la educación y del papel clave del educador en la sociedad. 


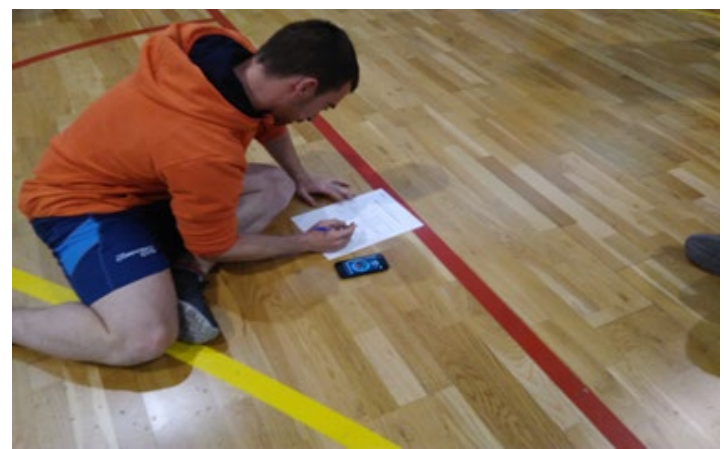

Fig. 1 Actividad práctica con el uso de las TAC

Las diferentes actividades prácticas promueven la participación del estudiante y lo convierten en el protagonista del proceso de enseñanza-aprendizaje. Se promueve el aprendizaje cooperativo para poder emprender un proyecto global donde cada contenido de la materia apoya en la parte del proyecto que está directamente relacionado con los diferentes apartados de éste.

Trabajar cooperativamente creando un proyecto educativo es un reto global para el estudiante y futuro profesor de Educación Física, y la integración de pedagogía, contenido y tecnología (Mishra y Koelher, 2006) en el desarrollo de un proyecto educativo dotan al alumno de una visión holística de los procesos de enseñanza y aprendizaje.

Se pretende que los futuros profesores sean capaces de diseñar propuestas concretas de intervención docente en los diferentes niveles de planificación educativa. También se fomenta el trabajo que vincule la teoría y la práctica, así como el desarrollo de actividades basadas en la evidencia científica que nos aportan las disciplinas afines a la Educación Física y al deporte y el conocimiento de las últimas tendencias de la enseñanza de la materia.

Se pretende profundizar en la búsqueda de los métodos y estilos de enseñanza más apropiados en función de los contenidos de aprendizaje, los objetivos didácticos y el perfil de los alumnos y grupos con los que se tenga que trabajar.

También es importante destacar la contribución al desarrollo de la competencia digital de los futuros docentes de la educación física con una actividad auténtica, funcional y aplicada.

En las sesiones teóricas y prácticas se recomienda que el alumno lleve dispositivos móviles con conexión a Internet (portátil, tableta o smartphone) para su uso atendiendo a las indicaciones del profesor.

A continuación de describen diferentes actividades desarrolladas en la materia y que responden a los objetivos que se plantean en el plan docente: el uso de MAV y las TAC (1), presentación de contenidos de la materia por parte de los alumnos en pequeños grupos (2), y participación en la organización de una actividad gamificada de promoción del deporte desde la Educación Física escolar con estudiantes reales de Educación Secundaria (3). 
Implementación de las Tecnologías de la Información y la Comunicación (TAC) en la materia Didáctica de la Educación Física en el Grado de Ciencias de la Actividad Física y el Deporte de la FPCEE Blanquerna - Universidad Ramon Llull

\subsection{Uso los MAV y las TAC}

Se trata de un proyecto por parejas con la realización de una presentación en línea que combine diferentes elementos dinámicos e interactivos.

La temática del proyecto versará sobre uno de los siguientes temas (tabla1):

Tabla 1. Temas a trabajar en el proyecto por parejas

\begin{tabular}{ll}
\hline \multicolumn{2}{c}{ Temas para el proyecto } \\
\hline $\begin{array}{l}\text { El papel de la actividad física y el deporte en la } \\
\text { mejora del rendimiento académico. }\end{array}$ & $\begin{array}{l}\text { El papel de la coeducación en la Educación } \\
\text { Física. }\end{array}$ \\
$\begin{array}{l}\text { El papel del profesor de Educación Física en la } \\
\text { motivación de sus alumnos. }\end{array}$ & $\begin{array}{l}\text { El tratamiento del cuerpo desde la Educación } \\
\text { Física. }\end{array}$ \\
$\begin{array}{l}\text { El papel de la Educación Física en la relación } \\
\text { con las otras materias. }\end{array}$ & El papel del cine como herramienta educativa. \\
$\begin{array}{l}\text { El papel de la Educación Física y el deporte en } \\
\text { la integración social. }\end{array}$ & $\begin{array}{l}\text { El papel de la Educación Física y el deporte como } \\
\text { formadores de líderes. }\end{array}$ \\
$\begin{array}{ll}\text { El papel de la Educación Física y el deporte en } \\
\text { la adquisición de valores. }\end{array}$ & $\begin{array}{l}\text { El papel de la Educación Física y el deporte como } \\
\text { prescriptores de hábitos saludables. }\end{array}$ \\
\hline
\end{tabular}

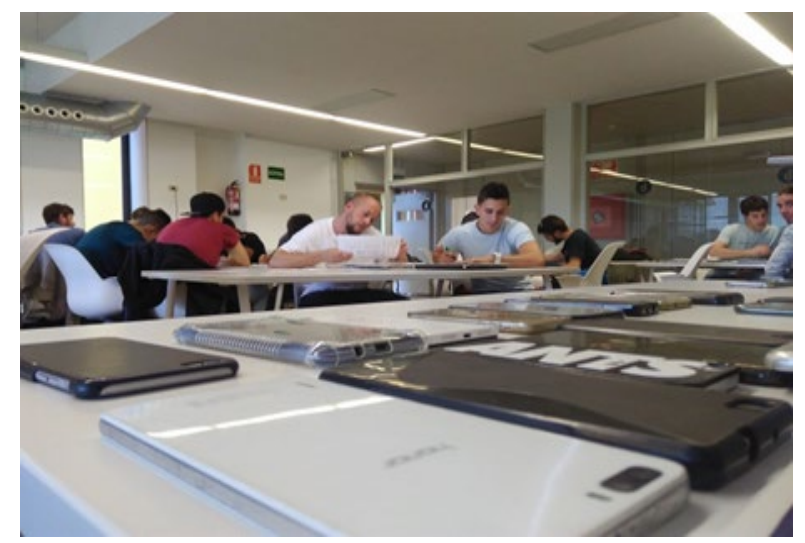

Fig. 2. Sesión de preparación del proyecto por parejas

El trabajo se entrega a través de un tuit en Twitter ${ }^{\circledR}$, que contendrá el título del trabajo, una imagen adjunta de alguna parte del proyecto y un enlace que redirige a una entrada de un blog creado con este fin.

La entrada del blog tendrá un título, una descripción que explique el trabajo y una presentación audiovisual en línea incrustada que desarrolla el tema con un vídeo de los componentes del grupo exponiendo los contenidos del proyecto asignado. 


\subsection{Presentación contenidos materia por parte de los alumnos en pequeños grupos}

El proyecto de desarrolla en grupos de 4-5 alumnos. Cada grupo debe realizar una presentación a los compañeros sobre un contenido de la materia propuesto por el profesor.

La presentación debe tener las siguientes características:

- Duración de diez minutos.

- Podrá ser con cualquier programa y/o aplicación diseñada para hacer presentaciones.

- Se valora la originalidad y la creatividad.

- La presentación deberá compartir en una carpeta de Google Drive ${ }^{\circledR}$ creada para este fin.

- Los temas a asignar son los siguientes (tabla 2):

Tabla 2. Temas a trabajar en el proyecto en grupos reducidos

\begin{tabular}{ll}
\hline \multicolumn{2}{c}{ Temas para el proyecto } \\
\hline Los estilos de enseñanza de instrucción directa. & El perfil del mejor profesor/a de Educación \\
& Física. \\
Los estilos de enseñanza de indagación. & Evaluaciones normativa y criterial. \\
Las estrategias en la práctica. & Instrumentos de evaluación en la Educación \\
& Física. \\
Atención a la diversidad. & ¿Qué esperan los alumnos de la Educación \\
& Física? \\
Tipos de sesiones en la Educación Física. & Las TIC y las TAC en la Educación Física. \\
\hline
\end{tabular}

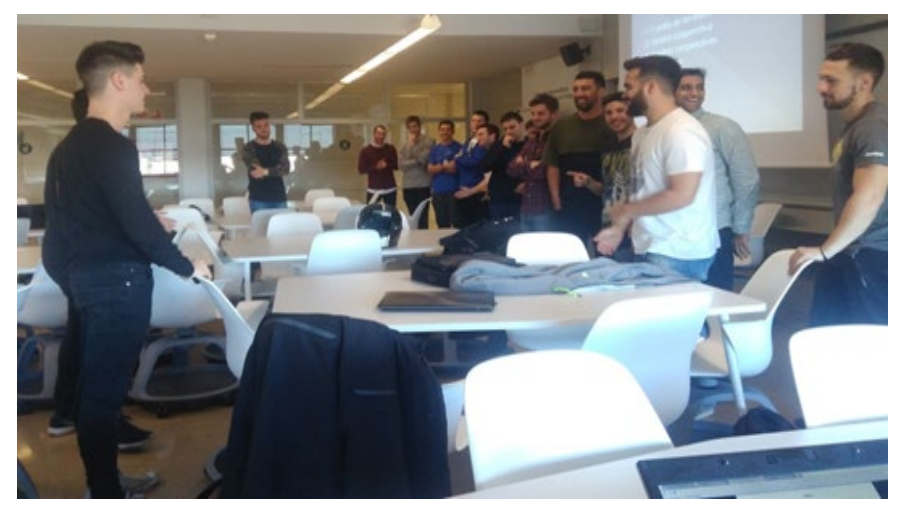

Fig. 3 Presentación dinámica del proyecto en pequeños grupos 
Implementación de las Tecnologías de la Información y la Comunicación (TAC) en la materia Didáctica de la Educación Física en el Grado de Ciencias de la Actividad Física y el Deporte de la FPCEE Blanquerna - Universidad Ramon Llull

\subsection{Participación en la organización de una actividad gamificada de promoción del deporte desde la EF escolar con estudiantes reales de Educación Secundaria.}

Este proyecto educativo se centra en la participación de los estudiantes en un programa gamificado de fomento de la actividad física y la adherencia al deporte por parte de estudiantes de Educación Secundaria Obligatoria.

Los contenidos teóricos de la propuesta abundan sobre el sedentarismo, los hábitos alimentarios, el estilo de vida de los adolescentes y cuáles son las herramientas al alcance del profesor de Educación Física para promover la actividad física y el deporte dentro y fuera del horario escolar.

En su parte práctica, el proyecto implementa una competición intercentros en la ciudad de l'Hospitalet de Llobregat (Barcelona) que agrupa 15 institutos de secundaria y que compiten durante todo el curso académico a través de actividades relacionadas con la práctica del CrossFit adaptadas a los alumnos.

Los estudiantes de la materia participan en la organización de la competición e interaccionan con los alumnos y con sus profesores durante su desarrollo.

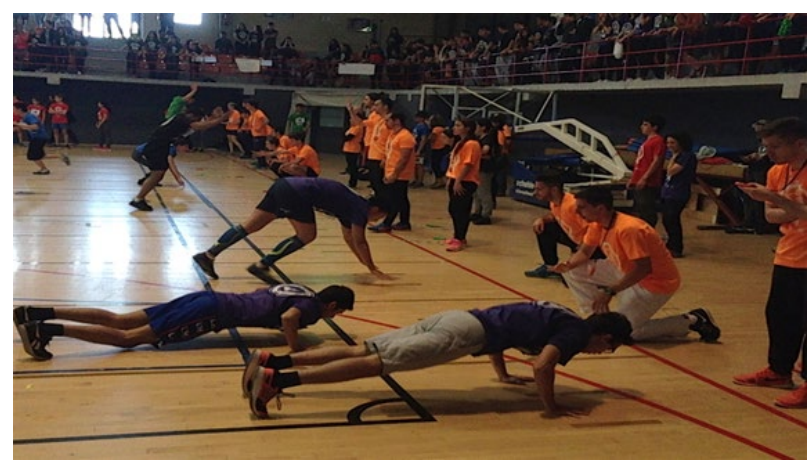

Fig. 4 Proyecto de Educación Física gamificada

\section{Resultados}

Un total de 150 estudiantes al año en los últimos ocho cursos académicos han participado de esta materia y se han podido impregnar de las metodologías planteadas y vividas.

Muchos de los blogs y cuentas de Twitter ${ }^{\circledR}$ que se han diseñado se han mantenido activos después de la participación en la materia, por lo tanto, la actividad de enseñanza-aprendizaje ha sido significativa y funcional a lo largo de los años.

Los estudiantes han utilizado diferentes e innovadoras herramientas para realizar presentaciones dinámicas, realizar encuestas en clase, monitorizar la actividad física en las clases de Educación Física (ver figura 5). 


\section{Google}

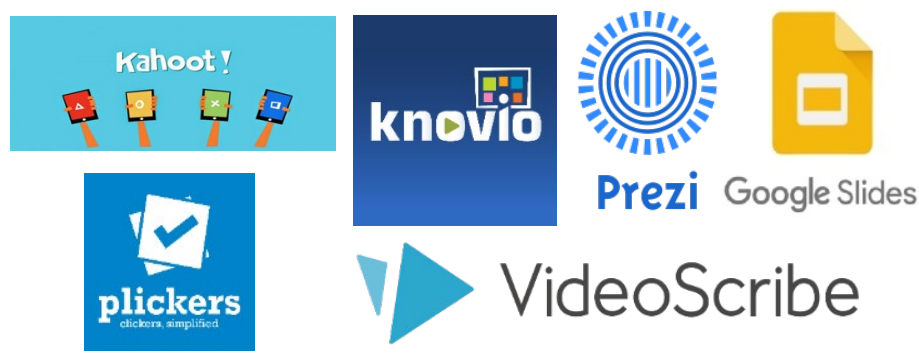

Fig. 5 Herramientas utilizadas por los estudiantes en la materia

En relación a la satisfacción de los estudiantes en relación con la materia y su desarrollo, los diferentes indicadores relativos a innovación, uso de diferentes metodologías, estructuración de los contenidos, recursos didácticos, etc. están por encima de los 8 puntos sobre 10, obteniendo una media de estos indicadores de 8,89 puntos, lo cual refuerza que se trata de una materia que gusta a los estudiantes y que les permite aprender de manera satisfactoria y real (tabla 3).

Tabla 3. Indicadores satisfacción estudiantes materia Didáctica de la Educación Física. Curso $2017 / 2018$

\section{Indicador de valoración}

Media

valoraciones

Las tareas previstas (teóricas, prácticas, de trabajo individual, en grupo, etc.) guardan relación con lo que el profesor pretende que aprenda en la actividad docente.

El profesor prepara, organiza y estructura bien las actividades o tareas que se realizan.

El profesor utiliza los recursos didácticos adecuados para facilitar el aprendizaje.

El profesor consigue despertar interés por los diferentes temas que se abordan en el desarrollo de la actividad docente.

El profesor favorece la participación del estudiante en el desarrollo de la actividad docente (facilita que exprese sus opiniones, incluye tareas individuales o de grupo, etc.).

He mejorado mi nivel de partida, en relación con las competencias previstas en el programa.

El profesor relaciona las actividades llevadas a cabo en la asignatura con los temas actuales.

El conocimiento del profesor, su metodología y el material que utiliza (bibliografía, actividades, ...) son innovadores, están actualizados y favorecen el proceso de aprendizaje. 
Implementación de las Tecnologías de la Información y la Comunicación (TAC) en la materia Didáctica de la Educación Física en el Grado de Ciencias de la Actividad Física y el Deporte de la FPCEE Blanquerna - Universidad Ramon Llull

Los comentarios de los estudiantes en relación al aprovechamiento y valoración de las actividades desarrolladas a lo largo de la materia, su utilidad e interés se resumen en la tabla 4.

Tabla 4. Comentarios de valoración de los estudiantes de la materia Didáctica de la Educación Física. Curso 2017/2018

\section{Comentarios de los estudiantes}

Clases de pequeño grupo con mucha diversidad de tareas.

Me ha servido mucho para mí futuro, ya que me quiero dedicar a los ámbitos docente y he visto muchos aspectos de los que en mí futuro aplicaré.

El profesor consigue aplicar nuevas metodologías y estrategias de aprendizaje innovadoras a sus sesiones que estimulan y mantienen la motivación de los alumnos. Ha sido una experiencia muy enriquecedora.

Despierta el interés del estudiante y fomenta la participación en todo momento.

Me ha gustado mucho la manera de dar clases que tiene el profesor; ya que con ironías, bromas, preguntas retóricas ... logra acercarse mucho al estudiante y crea un ambiente muy adecuada para seguir las clases.

Fomenta la participación de los alumnos mediante el uso de las TIC. Muy buen trabajo durante todo el semestre.

Fuente: Elaboración propia (2019)

\section{Conclusiones}

Las conclusiones de la aplicación de la propuesta se relacionan con el logro de los objetivos planteados en la materia, con el aprovechamiento por parte de los estudiantes y a través de la valoración de la satisfacción de éstos al final de cada curso académico.

Los objetivos propuestos en el proyecto se cumplen satisfactoriamente ya que los estudiantes desarrollan y mejoran su competencia digital, utilizan las TIC, las TAC y los MAV en las diferentes actividades, sean evaluables o no, y han conocido y utilizado diversas metodologías de enseñanza-aprendizaje aplicadas a la Educación Física. De esta manera, se contribuye al desarrollo de la competencia digital y al descubrimiento de herramientas útiles para la programación de actividades, de presentación de contenidos y de monitorización de la actividad física y deportiva de los estudiantes durante las clases.

Las valoraciones de los estudiantes al final de la materia demuestran que los aprendizajes son significativos, que guardan relación con la innovación y se facilita la participación a lo largo de todo el proceso, lo cual estimula el aprendizaje y la motivación del alumnado.

Año tras año, los profesores responsables de la materia incorporan nuevas actividades, herramientas y metodologías atendiendo a las diferentes tendencias e investigaciones al respecto con la voluntad de seguir innovando año a año.

A nivel de análisis futuro, muchos de los estudiantes han decidido seguir sus estudios cursando el Máster Universitario en Formación del Profesorado orientándose hacia el 
ejercicio profesional de la docencia de la Educación Física escolar y llevando a cabo algunas de las experiencias desarrolladas en la asignatura.

\section{Referencias}

ARÉVALO, M. (2016). Blog "La cajonera". http://lacajonerademarta.blogspot.com.es/p/tic-y-ef.html [Consulta: 10 marzo 2019]

CAMPOS-RIUS, J. y SEBASTIANI, E. M. (2016). "El repte de les TIC i les TAC en l’Educació Física" en Aloma: Revista de Psicologia, Ciències de l'Educació i de l'Esport, 34(2), 25-36.

CANALETA, X., VERNET, D. y NAVARRO, J. (2013). "Metodología on demand para el desarrollo de la asignatura de Sistemas Operativos Avanzados". En: Jornadas de Enseñanza Universitaria de la Informática (19.-2013: Castelló de la Plana). Universitat Jaume I. Escola Superior de Tecnologia i Ciències Experimentals.

CAPLLONCH, M. (2005). Las Tecnologías de la Información y la Comunicación en la Educación Física de Primaria: Estudio sobre sus posibilidades educativas. Tesis Doctoral: Barcelona. Universidad de Barcelona.

CARREIRO, C., GONZÁLEZ, M. A. y GONZÁLEZ, M. F. (2016). "Innovación en la formación del profesorado de educación física" en Retos: nuevas tendencias en educación física, deporte y recreación, $29,251-257$.

CONSELL ESCOLAR DE CATALUNYA (2015). Les tecnologies mòbils als centres educatius.

Generalitat de Catalunya.

$<$ http://consellescolarcat.gencat.cat/web/.content/consell escolar/actuacions/documents $\mathrm{i}$ informes e n_pdf/static_files/Doc1-15_Tecnologies_mobils.pdf $>$ [consulta: 1 marzo 2019]

FERRERES, C. (2011). La integración de las tecnologías de la información y de la comunicación en el área de la educación física de secundaria: análisis sobre el uso, nivel de conocimientos y actitudes hacia las TIC y de sus posibles aplicaciones educativas. Tesis Doctoral. Tarragona: Universitat Rovira i Virgili.

LLEIXÀ, T. (2017). "Didáctica de la Educación Física: Nuevos temas, nuevos contextos" en Didacticae: Revista de Investigación en Didácticas Específicas, 2, 2-5.

LÓPEZ, V., PÉREZ, D., MANRIQUE, J. y MONJAS, R. (2015). "Los retos de la Educación Física en el Siglo XXI" en Retos: nuevas tendencias en educación física, deporte y recreación, ,29, 182-187.

MISHRA, P. y KOEHLER, M. (2006). Technological Pedagogical Content Knowledge: A Framework for Teacher Knowledge. Michigan: Michigan State University.

PEIRE, T. y ESTRADA, J. (2015). "Innovación en la educación física y en el deporte escolar: Métodos de enseñanza, deportes y materiales alternativos" en E-balonmano. com: Revista de Ciencias del Deporte, 11(3), 223-224.

REIG, D. (2012). Socionomia. ¿Vas a perderte la revolución social? Barcelona: Deusto.

REIG, D. \& VÍLCHEZ, L. F. (2013). Los jóvenes en la era de la hiperconectividad: tendencias, claves y miradas. Madrid: Fundación Telefónica. 